
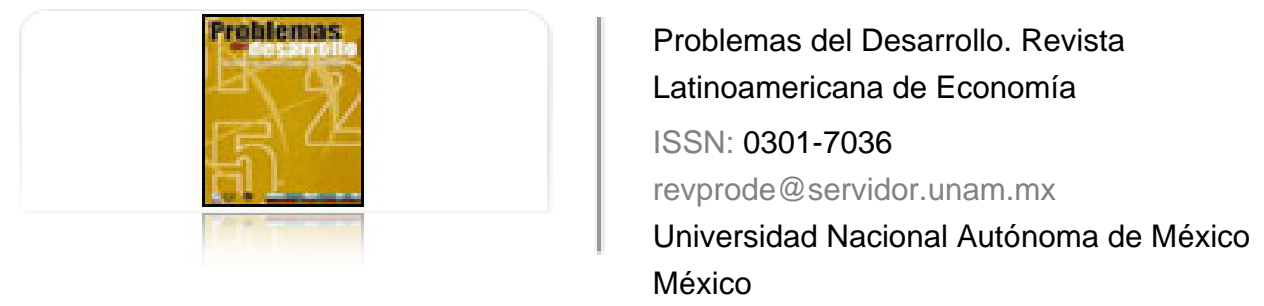

Trejo Nieto, Alejandra Berenice

Disparidades regionales en el sector manufacturero mexicano

Problemas del Desarrollo. Revista Latinoamericana de Economía, vol. 39, núm. 154, julio-septiembre, 2008, pp. 87-110

Universidad Nacional Autónoma de México

Distrito Federal, México

Disponible en: http://www.redalyc.org/articulo.oa?id=11820139005

Cómo citar el artículo

- Número completo

- Más información del artículo

Página de la revista en redalyc.org

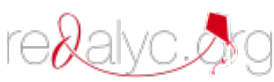

Sistema de Información Científica

Red de Revistas Científicas de América Latina, el Caribe, España y Portugal Proyecto académico sin fines de lucro, desarrollado bajo la iniciativa de acceso abierto 


\title{
DISPARIDADES REGIONALES EN EL SECTOR MANUFACTURERO MEXICANO
}

\author{
Alejandra Berenice Trejo Nieto*
}

Fecha de recepción: 11 de enero de 2008. Fecha de aceptación: 14 de marzo de 2008.

\section{Resumen}

Este artículo examina los cambios a nivel espacial del sector manufacturero mexicano. Las transformaciones ocurridas después de la liberalización comercial y el Tratado de Libre Comercio de América del Norte (TLCAN) son particularmente considerados. Empleamos información del Producto Interno Bruto (PIB) manufacturero (1970-2004) en el ámbito estatal en una revisión de participaciones y densidades estatales. Los datos revelan la existencia de un proceso de dispersión del PIB manufacturero total de largo plazo. A pesar de que se presentó una "mayor" dispersión geográfica después del programa de liberalización generalizada y después del TLCAN, la relocalización de la manufactura ya estaba en marcha. Los estados fronterizos con Estados Unidos (EU) fueron los beneficiarios iniciales de la descentralización pero las entidades del Bajío han tenido las alzas más grandes en las participaciones regionales dentro del PIB manufacturero recientemente. Sin embargo, no hay certeza de un cambio significativo en la estructura regional dual. La idea de causación acumulativa y la de la existencia de ventajas en las condiciones iniciales son adecuadas.

Palabras clave: disparidades regionales, manufactura, geografía económica, localización, apertura comercial.

* Profesora-investigadora de tiempo completo en el Centro de Investigaciones Socioeconómicas, Universidad Autónoma de Coahuila. México.Correo electrónico: atrejo@cise.uadec.mx. 


\section{Abstract}

This paper examines the changes in geographical patterns in Mexico's manufacturing sector. Particular attention is paid to the transformations that occurred after the trade liberalization and the North American Trade Agreement (NAFTA) was signed. We use information on manufacturing Gross Domestic Product (GDP) (1970-2004) by state, reviewing state participations and densities. The figures reveal the existence of a process of dispersion of total manufacturing gdp in the long term. Although a greater geographical dispersion presented itself after the generalized liberalization program and following the signing of the NAFTA, the relocation of manufacturing was already underway before that. The states bordering the United States were the initial beneficiaries of this decentralization, but the Bajio region states have recently had the largest increases in regional shares of manufacturing GDP. However, it is uncertain whether a significant change has taken place in the regional dual structure.

Key words: regional disparities, manufacturing, economic geography, location, trade opening.

\section{Résumé}

Cet article examine les changements survenus dans la répartition géographique du secteur manufacturier mexicain. Les transformations qui se sont produites après la libéralisation commerciale et le Traité de Libre Commerce d'Amérique du Nord (TLCAN) sont particulièrement prises en compte. Nous employons des données du produit intérieur brut de ce secteur (1970-2004) au niveau des différents états du pays, révisant leurs participations et densités. Celles-ci révèlent l'existence d'un processus de dispersion du produit manufacturier total sur le long terme. Bien que la dispersion géographique de la manufacture se soit révélée "plus importante" après le programme de libéralisation généralisée et le TLCAN, la délocalisation de la manufacture était déjà lancée. Les États frontaliers avec les États-Unis (EU) ont été, au début, les bénéficiaires de la décentralisation mais les états de la région centrale du Bajio ont eu récemment les hausses les plus importantes de participations régionales au PIB manufacturier. Cependant, il n'est pas certain que survienne un changement significatif dans la structure régionale duale.

Mots clés: disparités régionales, manufacture, géographie económique, localisation, ouverture commerciale.

\section{Resumo}

$O$ artigo estuda as mudanças a nível especial no setor manufatureiro mexicano. As transformações ocorridas depois da liberalização comercial e o Tratado de Livre Comércio da América do Norte (TLCAN) são particularmente consideradas. Empregamos informação do Produto Interno Bruto (PIB) manufatureiro (1970-2004) no âmbito estatal numa revisão de participações e densidades estatais. Os dados revelam a existência de um processo de dispersão do PIB manufatureiro total de longo prazo. A pesar de que se apresentou uma "maior" dispersão geográfica depois do programa de liberação generalizada e depois do TLCAN, a re-localização da manufatura já estava em andamento. Os estados fronteiriços com os Estados Unidos (EU) foram os beneficiados iniciais da descentralização, mas os estados do centro-oeste mexicano tiveram as maiores altas nas participações regionais dentro do PIB manufatureiro recentemente. No entanto, não há certeza de uma mudança significativa na estrutura regional de ambos.

Palavras chave: Desigualdades regionais, manufatura, geografia econômica, localização da apertura comercial. 


\section{Introducción}

n el análisis de la geografía económica al interior de los países se ha asumi-
do que las políticas proteccionistas condujeron a una creciente aglomeración
espacial de las actividades económicas, dado que en una economía cerrada al comercio externo las firmas tienden a localizarse cerca de su principal mercado doméstico — generalmente la capital del país—. En este sentido también se sugiere que los procesos de liberalización comercial y de integración económica tendieron a modificar los patrones de localización industrial. El escenario resultante — después de un shock externo como la liberalización comercial y la integración que ha tomado lugar - varía desde un panorama con una dispersión de la actividad económica hasta a uno con aglomeración creciente.

En realidad, la manera como puede modificarse la geografía económica de los países no es unilateral, sino dual porque la constituyen un par de aspectos:

a) Localización, es decir, el lugar donde la actividad económica decide ubicarse.

b) Aglomeración, que indica el grado de concentración de la actividad económica en determinado lugar.

En México, el dominio económico del Distrito Federal, seguido por algunos pocos estados, caracterizó la geografía de la manufactura durante el régimen de industrialización por sustitución de importaciones (ISI). En los años ochenta la economía mexicana se movió de una estrategia de industrialización orientada al mercado interno hacia un régimen de economía abierta. En gran parte, se anunció que las políticas de reestructuración de largo alcance serían benéficas en términos de equidad regional y de desarrollo espacial bajo la premisa de que las regiones atrasadas obtendrían ganancias de la creciente liberalización, y, como resultado, una estructura geográfica de la producción más homogénea y menos fragmentada. Sin embargo, ello aún es controversial. Contrario al argumento más tradicional, secundamos la idea de que el desarrollo espacial ha estado limitado y localizado en regiones específicas del país.

Investigaciones previas como las de Krugman y Livas Elizondo (1996), Chamboux-Leroux (2001), Sánchez-Reaza y Jordaan (2002), Corona Jíménez (2003), Decuir-Viruez (2003) y Sánchez-Reaza y Jordaan (2004) encontraron que una relocalización de la manufactura hacia el norte fue lo que caracterizó los ajustes geográficos que siguieron a la adopción del programa amplio de liberalización económica y comercial. Asimismo algunos de estos estudios también sugieren que el declive relativo del Distrito Federal ha sido menos claro con el paso del tiempo, debido a 
que esta región ha experimentado alguna recuperación durante algunos periodos. De ahí que dos preguntas permanezcan a discusión: 1) ¿en qué medida ha cambiado el patrón espacial de la producción en México a partir de la apertura económica? y 2) ¿cuál ha sido el papel de las reformas económicas en tal cambio?

A partir del conjunto de datos, este artículo intenta evaluar la cuestión de cómo la geografía económica de la manufactura mexicana ha evolucionado y se ha ajustado a partir de la liberalización y la integración económica.

Esta investigación sugiere que un impacto mínimo de tales shocks externos sobre la aglomeración — pero con un número significativo de cambios locacionales — es un escenario matizado que debe considerarse. Ciertamente, el resultado también depende de las condiciones iniciales, mientras que factores adicionales, aparte del comercio internacional y la inversión extranjera directa (IED), podrían llevar a uno u otro panorama.

Examinamos participaciones regionales y densidades así como indicadores sumarios de los niveles globales de aglomeración. El escrutinio de patrones pasados y actuales nos brinda la posibilidad de observar la dinámica de localización y aglomeración. Encontramos un número de hechos sobre estos aspectos, localización y aglomeración, que son las categorías analíticas empleadas para caracterizar el "paisaje geográfico" observado en la actividad manufacturera mexicana.

\section{Geografía económica interna y globalización económica}

Henderson et al. (2001) señalan la importancia de estudiar los aspectos espaciales de la economía debido a su conexión con los procesos de urbanización y la evolución del desarrollo regional. De forma similar, el papel de la globalización en la configuración del escenario geográfico de las economías nacionales y su desarrollo es considerado relevante. Al respecto dos transformaciones fundamentales en el contexto global también han modificado algunas perspectivas previas: en primer lugar, en la economía internacional —-mercados y producción —; en segundo, en las estrategias económicas nacionales de varios países de la industrialización por sustitución de importaciones hacia una industrialización orientada a las exportaciones (IOE).

En general, la globalización económica ha sido asociada con una creciente integración del comercio, la producción y los sistemas financieros entre países, y descrita como el proceso en el cual las transacciones económicas asumen un carácter transnacional. ${ }^{1}$

1 Hay una tendencia a destacar los aspectos económicos de la globalización; sin embargo, ésta involucra aspectos sociales, culturales, psicológicos, políticos, institucionales y ambientales. 
En este sentido, la globalización ha sido en parte producto de la apertura por medio de la liberalización comercial y la inversión extranjera, las cuales a su vez se han alcanzado mediante negociaciones multilaterales, esquemas de integración, acuerdos bilaterales y programas de liberalización unilaterales. Es probable que la globalización económica haya tenido algún efecto en la asignación espacial de la producción sobre su eficiencia de una forma similar a cómo ha influido en otros aspectos de la estructura económica y los parámetros de referencia más importantes de las actividades productivas. En esta situación, los territorios en sus diversas escalas experimentan transformaciones que llevan a procesos de ajuste a los nuevos escenarios internacionales.

De acuerdo con la teoría del comercio internacional tradicional, todas las formas de integración económica son benéficas en esencia. Pero la presencia de significantes economías externas y de rendimientos crecientes - la cual es resaltada por la nueva teoría del comercio internacional y la nueva geografía económica- podría causar que el comercio y la integración económica tuvieran un impacto adverso, como la desigual distribución de los beneficios por medio de regiones e industrias. Por ejemplo, regiones líderes tradicionales podrían capturar un porcentaje desproporcionado de las ganancias de la creciente liberalización, resultado de ventajas históricas. Esto se puede ejemplificar si trasladamos a un contexto multirregional la afirmación referente a un contexto internacional en el sentido de que un país con una fuerte posición inicial podría encontrarse a sí mismo con una ventaja que se acumula con el paso del tiempo (Krugman y Venables, 1996, p. 961).

Por otra parte, varios modelos teóricos tienen como escenario una estructura de centro-periferia que se refiere a un dualismo interno a los países. Típicamente las regiones atrasadas son agrícolas, mientras que las regiones desarrolladas albergan el grueso de la actividad manufacturera que trae los beneficios asociados de la industrialización.

Cuando la globalización económica lleva al establecimiento de regiones dinámicas, entendidas éstas como unidades geográficas subnacionales y las cuales son centros principales de actividad industrial, entonces sus efectos son geográficamente localizados, pues las regiones no se benefician de forma homogénea. En ese sentido, el efecto potencial de la globalización económica sobre la distribución interna de la actividad económica está relacionado con el desarrollo regional, además del desarrollo nacional. De hecho, los esfuerzos llevados a cabo de manera consistente por los gobiernos en los ámbitos nacional, regional o local para alcanzar el desarrollo económico se enfocan en atraer nueva inversión y expandir la ya existente, por lo que van

Vol. 39, núm. 154, julio-septiembre / 2008

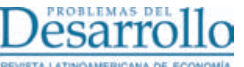


creando condiciones para localizar la actividad económica en sus territorios. De ahí que surja una relación entre geografía económica, desarrollo y globalización.

Mientras que la liberalización comercial y la inversión extranjera lograrían ofrecer oportunidades para el desarrollo de algunas regiones subnacionales, también podrían afectar de forma adversa a otras más. Por ejemplo, cuando hay cambios significativos en la localización de la actividad económica, a medida que la política de industrial va del proteccionismo hacia la promoción de exportaciones, entonces la liberalización y la integración económica causan shocks asimétricos, los cuales a su vez pueden tener impactos temporales o persistentes sobre la estructura espacial de la economía.

\section{Localización y aglomeración en las manufacturas}

Tradicionalmente, la aglomeración ha sido asociada con los agrupamientos de la actividad económica o poblacional en un lugar particular, sin que se hiciera explícita alguna demarcación de la escala geográfica o de la agregación industrial a la que el concepto se refiere. Sin embargo, la aglomeración es un concepto que requiere de algún tipo de delimitación. Para efectos de una mayor claridad analítica, realizamos una distinción entre aglomeración y localización, debido a que estos conceptos se tornan útiles en el análisis empírico. Nuestras definiciones son cercanas a las adoptadas por Brakman et al. (2001):

- Aglomeración geográfica: muestra la distribución espacial de la actividad económica a niveles agregados; por ejemplo, la manufactura total. Muestra cuánto o en qué medida los sectores agregados se localizan en un lugar particular.

- Localización: indica donde se ubican las actividades económicas o industrias en el espacio.

Las participaciones regionales se consideran un indicador detallado de la distribución completa de la economía a lo largo de los territorios. Además, brindan una descripción de los patrones de localización y aglomeración. Sin embargo, no provee una medida estricta de los niveles globales de agrupamiento económico. Para eso existen medidas que resumen en un solo número los niveles de aglomeración. De ahí que para hacer una primera aproximación a la distribución espacial de la manufactura, revisamos las participaciones regionales:

$$
\text { Ecuación 1: } S_{i}^{j}=s_{i j} / s_{n j}
$$


Donde $s_{i j}$ es un indicador del nivel de actividad económica (PIB, empleo, valor agregado, etcétera) de la región i en la industria j, y $s_{n j}$ es el monto en el ámbito nacional de la industria j. Una medida diferente de la distribución de la actividad económica son las densidades:

\section{Ecuación 2: $\mathrm{D}_{\mathrm{i}}=\mathrm{PIB}_{\mathrm{i}} / \mathrm{km}_{\mathrm{i}}{ }^{2}$}

Donde PIB $_{i}$ es el PIB manufacturero de la región i y $\mathrm{km}_{\mathrm{i}}^{2}$ es la superficie territorial de la región en kilómetros cuadrados. Los indicadores de densidad, los cuales indican el nivel de actividad económica por unidad de territorio — por ejemplo, producción o empleo por kilómetro cuadrado—-, son una buena aproximación de la distribución geográfica mediante la cual podemos controlar el efecto del tamaño de la región.

La investigación empírica que se ha centrado en los patrones y dinámica de la geografía económica emplea una gran variedad de indicadores sumarios de la aglomeración. Este análisis se basa en el Índice de Herfindahl y en una versión del Índice de Gini. El primero muestra el grado en que la actividad económica está distribuida por medio de un número de unidades geográficas. El valor máximo que toma es 1 , indicando el grado máximo de aglomeración (en una sola región); de manera alterna, valores cercanos a $1 / \mathrm{N}$ (donde $\mathrm{N}$ es el número de regiones) reflejan una alta dispersión de la actividad a lo largo de las regiones:

\section{Ecuación 3: $\mathrm{HERF}_{\mathrm{k}}=\sum_{\mathrm{n}=1}{ }^{\mathrm{N}}\left[\mathrm{Y}_{\mathrm{k}}{ }^{\mathrm{i}} / \mathrm{Y}_{\mathrm{k}}{ }^{\text {nacional }}\right]^{2}$}

Donde $\mathrm{Y}$ es el indicador de la actividad económica (producción, empleo, valor agregado); $k=$ industria; $N=$ número de regiones; $i=$ región.

Gordo et al. proponen una variante del Índice de Gini para medir la aglomeración absoluta. Este índice toma valores entre 0 y 1.

$$
\text { Ecuación 4: } \operatorname{GINI}_{\mathbf{k}}=\left(1 / 2 \mathbf{N}^{2}\right)(1 / \mu) \sum_{\mathrm{i}=1} \mathrm{~N} \sum_{\mathrm{j}=1}{ }^{\mathrm{N}}\left|\left(\mathbf{Y}_{\mathrm{k}}{ }^{\mathrm{i}} / \mathbf{Y}_{\mathrm{k}}{ }^{\text {Nacional }}\right)-\left(\mathbf{Y}_{\mathbf{k}}{ }^{\mathrm{j} / \mathbf{Y}_{\mathrm{k}}}{ }^{\text {Nacional }}\right)\right|
$$

Donde $\mu$ es la media simple de las participaciones regionales.

Se obtuvieron datos del PIB manufacturero en valores absolutos y participaciones regionales de bases de datos en línea del Instituto Nacional de Estadística, Geografía e Informática (INEGI). Información estatal de varios años está disponible en los niveles de uno y dos dígitos. Se pueden obtener datos anuales entre 1993 y 2004, pero sólo se dispone de datos quinquenales en el periodo 1970-1985.

Vol. 39, núm. 154, julio-septiembre / 2008

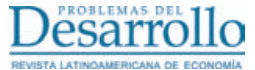


ALEJANDRA BERENICE TREJO NIETO

\section{La vieja geografía de la manufactura mexicana}

En casi tres décadas - a partir de los años cincuenta y hasta los setenta-México, como muchas economías de nueva industrialización (ENI), siguió una estrategia de sustitución de importaciones para promover el desarrollo industrial y económico. A partir de la liberalización comercial de inicios de los ochenta, la promoción de las exportaciones junto con la privatización y la desregulación de la economía fueron el esquema general para fomentar el desarrollo económico. En la práctica, la apertura económica ha tomado la forma de una integración comercial principalmente con Norteamérica. El dominio del Distrito federal (DF) durante el periodo proteccionista es un aspecto bien conocido de la geografía manufacturera. En 1970 esta región generó más de 32\% del PIB manufacturero nacional, mientras que Jalisco, Estado de México (EM) y Nuevo León (NL) junto con el DF generaron más de 66\%. Ello representó un fenómeno particular porque estas regiones abarcan sólo 12\% de la superficie del país. Esto indica una alta aglomeración geográfica de actividad manufacturera en el DF, y en una menor medida en el EM, Jalisco y NL durante la etapa de economía cerrada. Al principio de los años ochenta esta situación no había cambiado, pues sólo se presentó una ligera disminución en la participación de estas regiones. Sobrino (2003) encuentra que el DF generó 30\% del PIB, el EM 19\%, NL 10\% y Jalisco 7\%. SánchezReaza y Jordaan (2002) y Sánchez-Reaza y Jordaan (2004) encuentran que alrededor de $45 \%$ de empleo total manufacturero se localizaba en EM y DF al inicio de los años ochenta. Estos autores plantean que esta aglomeración en el DF y el EM se dio debido a un acelerado proceso de urbanización y a los consecuentes flujos migratorios de las áreas rurales a las urbanas. Otra proporción relativamente importante de la actividad manufacturera se ubicaba en NL y Jalisco, que albergan las otras dos áreas metropolitanas más grandes del país, Monterrey y Guadalajara. En contraste, el resto de los estados experimentó una aglomeración industrial de ligera a pobre durante esta etapa. Sin embargo, a pesar de que Estado de México, Jalisco y Nuevo León tuvieron participaciones considerables, estas entidades permanecieron muy lejos del DF.

Respecto a la distribución espacial de la manufactura después de la liberalización comercial, Corona Jiménez encuentra que la participación de la capital del país dentro de las empresas manufactureras más grandes fue la más alta, seguida también por la del EM, NL y Jalisco. Aunque ocurrió cierta dispersión geográfica, el proceso no fue lineal. Entre 1980 y 1989 las participaciones del DF disminuyeron, mientras que las del EM y NL también descendieron, pero en menor medida. Entre 1986 y 1990 los estados con los aumentos más importantes fueron las regiones norteñas de Coahuila, Chihuahua, Sonora, Tamaulipas y Baja California, aunque

\section{Desarrarrollo}


la participación de estados en el Bajío como San Luis Potosí y Guanajuato también aumentó. En el periodo 1990-1999 los principales incrementos se dieron en las entidades norteñas además de DF y Jalisco. Sánchez-Reaza y Jordaan (2002) y Sánchez-Reaza y Jordaan (2004) observan que la participación de DF en el empleo disminuyó de manera progresiva. En contraste, la participación de estados fronterizos como Sonora, Chihuahua y Coahuila mejoraron visiblemente. Ello muestra un proceso de localización/relocalización del empleo hacia el norte. De forma similar, en su análisis de las participaciones regionales, Chamboux-Leroux encuentra que entre 1985 y 1998 el grupo de estados fronterizos aumentó su participación en $11 \%$, mientras que las regiones del centro perdieron $10 \%$. Por otra parte, entre 1988 y 1998, 73\% de nuevos empleos manufactureros fue generado en sólo 10 entidades, de las cuales seis eran norteñas y cuatro estaban en el centro y el Bajío. Sin embargo, según Decuir-Viruez, la descentralización industrial consistió en el movimiento de la actividad económica no sólo hacia la frontera, sino también al EM y otros estados vecinos; es decir, fue un desplazamiento desde el DF a su periferia cercana. Asimismo, Sobrino comenta que, aunque experimentaron una contracción en su participación, el DF y el EM conservaron su lugar como los principales generadores de valor agregado industrial. Jalisco y NL permanecieron en los primeros lugares, mientras que Coahuila, Chihuahua y Puebla completaron la lista de estados con las participaciones más altas en la actividad industrial. Ello significa que ocurrió una dispersión localizada de la manufactura. Corona Jíménez señala que el declive relativo del DF no ha sido estable. Entre 1986 y 1990 la participación de esta región decreció, pero en el periodo 1990-1999, y particularmente en 1994, la tendencia se revertió y el DF se repuso. Esta recuperación se frenó en 1999 cuando su participación disminuyó nuevamente.

\section{Dinámica de la geografía manufacturera: movimientos locacionales y aglomeración persistente}

De acuerdo con el análisis de las participaciones regionales, durante el periodo inicial del programa de liberalización comercial generalizada (1980- 1985), en el PIB manufacturero se encuentra lo siguiente:

1. La participación del DF, la región industrial dominante, se contrajo en casi $4 \%$ pasando de 29\% a 25\%. Las otras regiones importantes Jalisco, EM y NL tuvieron pequeños incrementos en sus participaciones. Con ello se dio una disminución conjunta de las cuatro regiones dominantes de aproximadamente $2.5 \%$.

Vol. 39, núm. 154, julio-septiembre / 2008

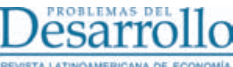


2. El comportamiento de los estados en la frontera con EU, quitando NL, no fue uniforme; las participaciones de Chihuahua, Coahuila, y Sonora crecieron mientras que las de Tamaulipas y Baja California disminuyeron. Por ello tuvieron en conjunto un aumento aunque marginal $(0.75 \%)$.

3. Las participaciones de todos los estados a lo largo del corredor formado por Aguascalientes, San Luis Potosí, Querétaro y Guanajuato aumentaron, con un incremento agregado de $2.4 \%$.

4. Los estados en el sur y la península de Yucatán, excepto Quintana Roo, disminuyeron su contribución al PIB manufacturero.

Sin embargo, por lo menos, algunas de estas tendencias datan de los años setenta:

1. La participación del DF cayó de 32\% a $29 \%$ entre 1970 y 1980.

2. El EM presentó un ligero incremento.

3. Coahuila y Chihuahua presentaban participaciones crecientes mientras que la de Baja California declinó.

4. Los estados del Bajío, a excepción de Guanajuato, mejoraron sus participaciones.

5. En el sur, Campeche, Guerrero y Yucatán experimentaron caídas de varios grados.

Un hecho destacado es que Chiapas y Oaxaca tuvieron aumentos probablemente por la expansión de un número de industrias ligadas al procesamiento de petróleo. Sin embargo, así como el auge petrolero de finales de los años setenta, el dinamismo repentino de estas industrias fue temporal.

A partir de la entrada del Tratado de Libre Comercio con Norte América (TLCAN) en 1994 y hasta 2004, observamos:

1. Una participación decreciente de DF (excepto en los periodos 1996-1997 y 2001-2002), así como del EM y Jalisco además de una participación en aumento de Nuevo León después de 1996.

2. Incrementos irregulares en la mayoría de los estados fronterizos del norte con una mejora conjunta de $3 \%$.

3. Una tendencia creciente y constante de las partes en participaciones de los estados del Bajío, sobre todo en Querétaro y Guanajuato.

4. Por otro lado, en el sur todos los estados permanecieron con participaciones marginales, las cuales además presentaron una tendencia decreciente (excepto Yucatán con pequeños aumentos).

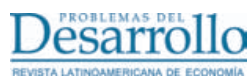

Vol. 39, núm. 154, julio-septiembre / 2008 
Aunque el DF presentó una progresiva baja en su participación en el PIB manufacturero y otros estados se vieron beneficiados por tal proceso, las directrices ya mencionadas nos dicen poco sobre el grado en el cual los niveles de aglomeración como tal han cambiado en el tiempo. Con una metodología similar a la utilizada en el análisis de convergencia del ingreso per capita hacemos una regresión, teniendo como variable dependiente el cambio relativo en las participaciones regionales en el PIB manufacturero entre dos periodos contra las participaciones en el lapso inicial para tener una idea de los cambios en la aglomeración en tal periodo. Si el coeficiente en las participaciones iniciales es negativo, ello indicará un proceso en el que las diferencias en las participaciones regionales disminuyan, lo que se podría interpretar como una reducción en la aglomeración.

El cuadro 1 muestra un resumen de las estimaciones para dos periodos, 1970-1985 y 1993-2004.

Cuadro 1

Convergencia en las participaciones estatales

\begin{tabular}{lcc}
\hline Variable & Cambio 1985-1970 & Cambio 2004-1993 \\
\hline Constante & 1.2855 & 9.3790 \\
& $(0.1050)$ & $(0.5102)$ \\
SGDP + t- & -0.0183 & -0.0289 \\
& $(0.0150)$ & $(0.0915)$ \\
Adjusted R2 & 0.046 & 0.0033 \\
No. of obs & 32 & 32 \\
\hline
\end{tabular}

Errores estándar entre paréntesis

Las estimaciones arrojan un coeficiente negativo, lo que indica que las participaciones regionales tendieron a converger entre 1970 y 1985 y entre 1993 y 2004. Sin embargo, los coeficientes no son estadísticamente significativos y los $\mathrm{R}^{2}$ son cercanos a cero. Si la evolución de los niveles de aglomeración coincide con la aglomeración decreciente que sugiere el ejercicio de análisis de convergencia a continuación revisamos algunos índices sumarios.

Primero presentamos los cambios en la desviación de estándar (DE) de las participaciones regionales. Los cambios ascendentes en la DE indican un aumento en las disparidades de las participaciones regionales, mientras que valores más bajos en la 
DE significan una distribución más homogénea. ${ }^{2}$ Los cálculos de las DE revelan una tendencia de largo plazo de disparidades decrecientes. Hay aumentos en la DE únicamente en 1997 y 2002 que indican variaciones al alza en la aglomeración en esos dos años (cuadro 2).

Cuadro 2

Desviación estándar de las participaciones regionales

\begin{tabular}{ccc}
\hline Año & Desviación estándar & Resultado \\
\hline 1970 & 4.9207 & NA \\
1975 & 4.7350 & Dispersión \\
1980 & 4.6040 & Dispersión \\
1985 & 3.9298 & Dispersión \\
\hline 1993 & 4.692 & NA \\
1994 & 4.585 & Dispersión \\
1995 & 4.433 & Dispersión \\
1996 & 4.351 & Dispersión \\
1997 & 4.397 & Aglomeración \\
1998 & 4.356 & Dispersión \\
1999 & 4.337 & Dispersión \\
2000 & 4.327 & Dispersión \\
2001 & 4.314 & Dispersión \\
2002 & 4.320 & Aglomeración \\
2003 & 4.211 & Dispersión \\
2004 & 4.195 & Dispersión \\
\hline
\end{tabular}

NA: No aplica

Fuente: Cálculos propios.

De forma similar, el índice de Herfindhal (cuadro 3) muestra que los niveles de aglomeración disminuyeron entre 1970 y 1985; sin embargo, las reducciones nunca fueron dramáticas entre periodos, al presentarse la caída más grande entre

2 Aumentos de la desviación estándar indican una ampliación de las diferencias en los valores de la distribución muestral. Es decir, la DE más grande muestra que existe más disparidad entre observaciones. Por lo tanto, una disminución de la DE indica que la distribución se vuelve más homogénea. En el caso de las participaciones regionales una disminución de la DE probablemente indica que el PIB se redistribuye entre un mayor número de regiones (dispersión).

\section{Desaarrollo}

Vol. 39, núm. 154, julio-septiembre / 2008 
1980 y 1985. De 1993 en adelante se notan unos niveles más constantes; aunque la aglomeración continuó con una tendencia a la baja, no se presentaron variaciones sustanciales. El cambio más extenso ocurrió entre 1994 y 1995, el periodo inmediato después de que el TLCAN entrara en vigor. Los incrementos en la aglomeración se observaron sólo en 1997 y 2002.

Cuadro 3

Índice Herfindhal de aglomeración de la producción manufacturera

\begin{tabular}{lcc}
\hline Año & Índice & Resultado \\
\hline 1970 & 0.1558 & NA \\
1975 & 0.1416 & Dispersión \\
1980 & 0.1408 & Dispersión \\
1985 & 0.1215 & Dispersión \\
\hline 1993 & 0.0995 & NA \\
1994 & 0.0964 & Dispersión \\
1995 & 0.0922 & Dispersión \\
1996 & 0.0899 & Dispersión \\
1997 & 0.0912 & Aglomeración \\
1998 & 0.0901 & Dispersión \\
1999 & 0.0895 & Dispersión \\
2000 & 0.0893 & Dispersión \\
2001 & 0.0889 & Dispersión \\
2002 & 0.0891 & Aglomeración \\
2003 & 0.0862 & Dispersión \\
2004 & 0.0831 & Dispersión \\
\hline
\end{tabular}

Fuente: Cálculos propios.

Ambos indicadores, la DE y el índice de Herfindhal, muestran que la aglomeración aumenta en 1997 y 2002, la dispersión es la tendencia general y los cambios, sin embargo, no son dramáticos. ¿Cómo se explican cambios tan pequeños en los niveles de aglomeración? Dos factores pueden explicarlo: por un lado, las características de los índices; por otro, Sobrino señala una contracción de menor importancia del DF lo que ha garantizado su supremacía sostenida (causalidad acumulativa y condiciones iniciales). Las participaciones del DF se recuperan justo en los periodos en los que el grado de aglomeración aumenta (1997 y 2002), mientras que las reducciones posteriores a 1998 son muy pequeñas. 
El índice de Gini produce resultados parecidos (cuadro 4):

1) Existe aglomeración con tendencia decreciente, habiendo una disminución global entre 1970 y 1985 y entre 1993 y 2004.

2) A pesar de la existencia de movimientos anuales al alza y a la baja no hay grandes variaciones de un año a otro.

Cuadro 4

Índice Gini de aglomeración

\begin{tabular}{ll}
\hline Año & Índice \\
\hline 1970 & 0.6999 \\
1975 & 0.6807 \\
1980 & 0.6791 \\
1985 & 0.6557 \\
\hline 1993 & 0.6207 \\
1994 & 0.6098 \\
1995 & 0.6156 \\
1996 & 0.6051 \\
1997 & 0.5510 \\
1998 & 0.6104 \\
1999 & 0.6116 \\
2000 & 0.6109 \\
2001 & 0.5944 \\
2002 & 0.6129 \\
2003 & 0.6068 \\
2004 & 0.6007 \\
\hline
\end{tabular}

Fuente: Cálculos propios.

Todos los indicadores coinciden con la existencia de dispersión de largo plazo. La diferencia es que mientras que el índice de Gini demuestra un aumento en la aglomeración en el año subsecuente al TLCAN el índice de Herfindhal muestra dispersión. Aunque la mayor dispersión se presenta inmediatamente después de la puesta en marcha de las principales reformas comerciales, los niveles de aglomeración tendieron a estabilizarse. Después de la liberalización comercial la localización industrial persistió con un movimiento hacia el norte y el Bajío — pero en mayor medida hacia este último—. A la postre la aglomeración industrial parece consolidarse en localizaciones industriales tradicionales y en regiones concretas beneficiadas por la dispersión industrial.

\section{Desaarrollo}

Vol. 39, núm. 154, julio-septiembre / 2008 
Estos resultados apoyan la observación de Corona Jiménez de que las políticas de liberalización impulsaron un ajuste que implicó la relocalización a otros estados y una caída del DF. Sin embargo, con cada nueva reforma los cambios subsecuentes fueron más pequeños y los movimientos hacia la frontera se tornaron menos probables. En cambio, lo que aumentó fue una relocalización hacia estados del Bajío, además de la nula existencia de movimientos importantes hacia regiones en el sur. Ello se traduce a una aglomeración persistente pero desarrollándose en localizaciones alternas; en el mismo sentido se observa que la relativa caída de algunos de los estados tradicionalmente industrializados ha favorecido solamente a estados en la frontera y en el Bajío.

Indudablemente se presenta una localización y relocalización de la producción manufacturera desde el DF a otros estados, lo cual confirma lo encontrado en estudios anteriores.

Sin embargo, lo que hay que resaltar acerca de la revisión anterior se resume en un par de hechos particulares:

1) Una contraída aunque persistente aglomeración de la actividad manufacturera ha estado aconteciendo. En general, los niveles de aglomeración muestran variaciones exiguas y el DF sigue sosteniendo su predominio en términos absolutos.

2) El proceso de dispersión estaba en marcha antes de la liberalización comercial. Los estados en la frontera con EU comenzaron a edificar una base industrial importante desde los años sesenta, mientras que los estados en el Bajío también comenzaron a gozar en cierta medida de la dispersión manufacturera previamente a la liberalización comercial generalizada.

\section{Una visión alternativa: indicadores de densidad}

Las densidades de la producción son otra manera de abordar las diferencias regionales y observar si existe una distribución geográfica de la actividad económica desequilibrada en relación con las superficies territoriales de los estados. Las diferencias regionales también se manifiestan al tener en cuenta tamaños regionales pues las densidades varían perceptiblemente de estado a estado (mapa 1). Los dos extremos son DF y Baja California Sur, con casi 37,191 mil y 5 mil pesos mexicanos por kilómetro cuadrado respectivamente en 2000. DF, EM, Morelos y Aguascalientes han tenido las densidades más grandes de la producción y han ocupado por lo general las mismas posiciones entre 1993-2004. Los 10 estados con mayores densidades se ubican sobre todo en el centro del país y en el Bajío, a excepción de NL que está 
en la frontera norte pero ha sido parte de los núcleos industriales tradicionales. Otros estados en el norte tienen densidades medianas, mientras que en el sur hay primordialmente estados con baja densidad. Por otra parte, no se presentaron cambios significativos en las posiciones relativas de los estados entre 1993 y 2004.

Mapa 1

Densidades en el PIB manufacturero por estado

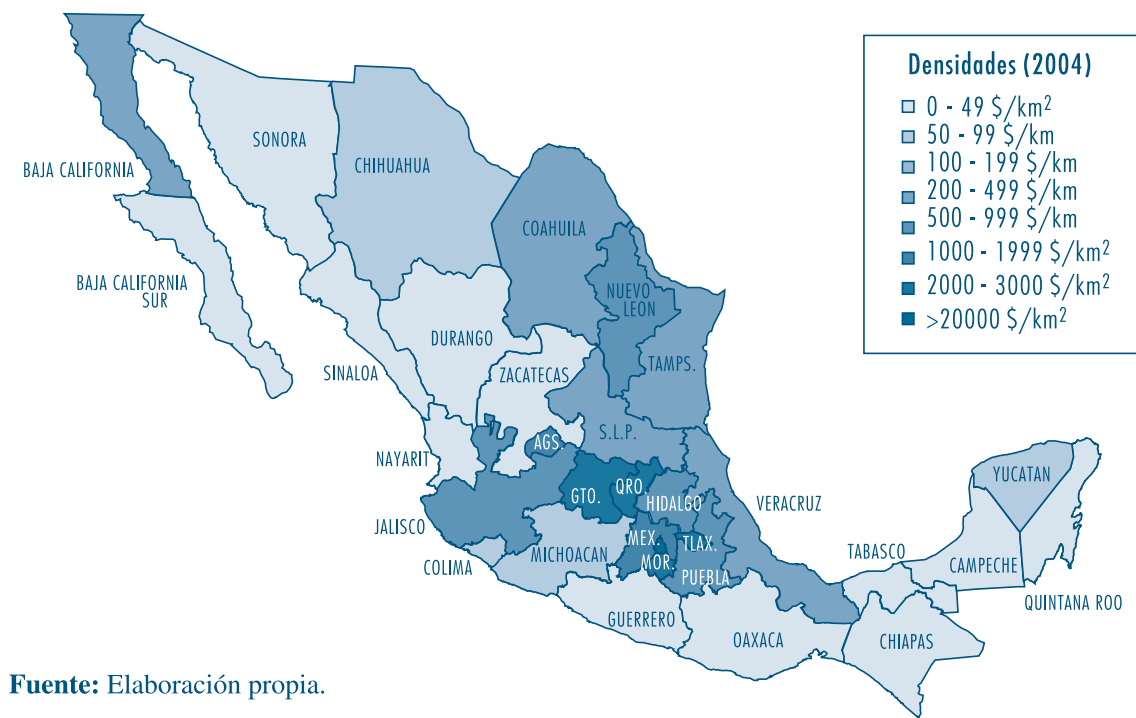

El coeficiente de variación de las densidades del PIB manufacturero indica que al considerar el tamaño territorial de los estados la tendencia que gobierna es la dispersión de la producción entre regiones. Hay una aglomeración creciente en 1997, pero en contraste con la DE de las participaciones regionales y del índice de Herfindhal este incremento también ocurrió en 1996 y 2000, pero no en 2002 (cuadro 5). A pesar de esto, los resultados no muestran que se haya alcanzado una igualación regional sustancial como resultado de la liberalización comercial o que la dispersión haya sido extensa.

En un estudio de la geografía económica de España, Brand encuentra que la liberalización comercial dio lugar a una reorganización de estructuras industriales en las aglomeraciones manufactureras españolas. En ese caso la liberalización y la integración comercial creciente no fueron lo suficientemente intensas para minar la estructura geográfica existente de la producción. De forma similar, una importante 
Cuadro 5

Coeficiente de variación de las densidades de producción

\begin{tabular}{lcc}
\hline Año & Coeficiente de variación & Resultado \\
\hline 1993 & 1.5274 & NA \\
1994 & 1.4943 & Dispersión \\
1995 & 1.4438 & Dispersión \\
1996 & 1.4593 & Aglomeración \\
1997 & 1.4792 & Aglomeración \\
1998 & 1.4684 & Dispersión \\
1999 & 1.4681 & Dispersión \\
2000 & 1.4752 & Aglomeración \\
2001 & 1.4697 & Dispersión \\
2002 & 1.3821 & Dispersión \\
2003 & 1.3476 & Dispersión \\
2004 & 1.3095 & Dispersión \\
\hline
\end{tabular}

Fuentes: Cálculos propios.

proposición acerca de la geografía económica mexicana y del ajuste posterior a la liberalización comercial y el TLCAN surge en este sentido.

Una decreciente pero persistente aglomeración del sector manufacturero mexicano es evidencia de que la integración no la ha afectado de forma sustancial, sino que sólo ha alterado algunos patrones locacionales.

Además, como el predominio del DF parece perenne, el argumento referente a la presencia de procesos de causalidad acumulativa y el de las ventajas iniciales se refuerza en el contexto intranacional donde la supremacía del DF se ha mantenido con el tiempo.

\section{Esbozo de una configuración espacial de la producción manufacturera}

\section{Una estructura regional dual}

La respuesta a la cuestión de si hay una estructura regional dual en México, al estilo del modelo centro-periferia, y si esa estructura ha cambiado con respecto a etapas anteriores es debatible y controversial. Por un lado está el problema de definir las características que una localización debe poseer para ser incluida en uno u otro grupo regional. Por otra parte, está la cuestión de la homogeneidad. ¿El centro está formado por una gran región homogénea con una estructura y dinámica de la producción única? $¿ \mathrm{O}$, por el contrario, puede el centro incluir varias regiones dinámicas que cohabitan 
geográficamente con centros secundarios? ¿Existe la posibilidad de dualidad dentro del centro o dentro de la periferia? Respuestas directas a tales cuestionamientos no son fáciles de formular sobre todo porque en general nos enfrentamos con diversas definiciones, agrupaciones regionales, interpretaciones y conclusiones.

El patrón geográfico dual de la economía mexicana basado en la noción de centroperiferia es una de tantas opciones plausibles al caracterizar la estructura regional. Rodríguez-Posse y Sánchez-Reaza definen el patrón territorial interno durante el periodo de ISI como economía dual. Bajo el modelo de sustitución de importaciones, dicen, la actividad industrial se aglomeró en el centro del país, pero también emergieron sitios de producción industrial en los estados fronterizos del norte. En el contrario, poca actividad industrial se localizó en los estados del sur. También sugieren que este patrón emergió antes del modelo proteccionista. Es por ello que se requiere de precaución al emitir afirmaciones tales como la referente a que la formación de los centros metropolitanos urbanos e industriales como la ciudad de México fueron el "subproducto involuntario" de las políticas de industrialización hacia adentro. Es decir, las características intrínsecas del modelo sustitutivo acentuaron mas no crearon el atraso económico de los estados del sur, los cuales permanecieron relativamente aislados de todo el proceso industrializador y dependientes sobre todo de las actividades primarias. Asimismo, el modelo de SI mantuvo y reforzó los centros metropolitanos ya existentes, los cuales albergaron los principales polos industriales y fueron además los mercados relevantes para las industrias nacionalmente protegidas. El surgimiento de la industria maquiladora de exportación en los sesenta contribuyó a la configuración de un patrón espacial en el cual las regiones en la frontera norte fueron incorporadas en un proceso de la industrialización puesto que este programa favoreció la localización de cierta actividad industrial.

Con el mismo concepto de dualidad, Bassols Batalla (1998) sugiere que seis de ocho regiones han avanzado hacia una integración relativamente alta entre ellas, por tanto con tendencia a la formación de un centro consolidado. ${ }^{3} \mathrm{Al}$ conservar la continuidad geográfica el centro del modelo dual consistiría en el área que va del centro del país a la frontera con EU. En contraste, la periferia serían los estados del sur incluyendo la península de Yucatán. En palabras de Bassols, la estructura dual fue originada

3 Baja California, Baja California Sur, Sonora, Sinaloa y Nayarit en el noroeste; Chihuahua, Coahuila, Durango, Zacatecas y San Luis Potosí en el norte; NL y Tamaulipas en el noreste; Jal., Aguascalientes, Colima, Michoacán y Guanajuato en el oeste; Querétaro, EM, DF, Morelos, Hidalgo, Tlaxcala y Puebla en el centroeste; Veracruz y Tabasco en el este. Los estados restantes Guerrero, Oaxaca y Chiapas en el sur y Campeche, Yucatán y Quintana Roo en la península de Yucatán. 
por un proceso caótico de desarrollo de muchos años, en el cual dos amplias regiones económicas subsistieron. Debido a la manera particular en la cual se fue dando el proceso del desarrollo económico, una de esas regiones asumió la supremacía, concedida históricamente incluso antes de que los españoles conquistaran el país.

Si reunimos los 32 estados en grupos más amplios tenemos una perspectiva más clara de estructuras regionales alternas. Por ejemplo, las regiones en la frontera norte (con excepción de NL), esto es, Baja California, Chihuahua, Coahuila, Sonora y Tamaulipas, y las regiones industrializadas tradicionales, DF, EM, Jalisco y Nuevo León, conforman una sola región denominada "centro" y se comparan con la región formada por los estados del sur y la península de Yucatán, la "periferia".

En el periodo 1970-2003 hay una tendencia a la baja en el centro, explicada sobre todo por caídas en las participaciones del DF. Sin embargo, las participaciones de las regiones atrasadas de la periferia también disminuyeron en ese lapso, indicando que de ninguna forma la caída del DF favoreció la localización industrial en el sur (cuadro 6). Al aglutinar al resto de los estados dentro del "centro" y compararlos con los del sur y la península de Yucatán, la distribución parece haber cambiado poco desde 1970 (cuadro 7).

Cuadro 6

Participaciones regionales en el PIB manufacturero 1970-2003

\begin{tabular}{ccc}
\hline Año & Norte y centro & Periferia \\
\hline 1970 & 75.87 & 3.4 \\
1975 & 73.87 & 3.76 \\
1980 & 73.09 & 3.71 \\
1985 & 71.51 & 2.98 \\
\hline 1993 & 70.81 & 3.18 \\
1994 & 70.22 & 3.19 \\
1995 & 69.83 & 3.31 \\
1996 & 69.46 & 3.17 \\
1997 & 69.94 & 2.86 \\
1998 & 69.62 & 2.87 \\
1999 & 69.67 & 2.8 \\
2000 & 69.76 & 2.8 \\
2001 & 69.25 & 2.94 \\
2002 & 69.02 & 2.94 \\
2003 & 68.45 & 3.01 \\
2004 & 69.07 & 2.96 \\
\hline
\end{tabular}

Fuente: Cálculos propios. 
Cuadro 7

Participaciones del centro-periferia en el PIB manufacturero 1970-2004

\begin{tabular}{lll}
\hline Año & Centro & Periferia \\
\hline 1970 & 96.60 & 3.4 \\
1975 & 96.24 & 3.76 \\
1980 & 96.29 & 3.71 \\
1985 & 97.02 & 2.98 \\
\hline 1993 & 96.82 & 3.18 \\
1994 & 96.81 & 3.19 \\
1995 & 96.69 & 3.31 \\
1996 & 96.83 & 3.17 \\
1997 & 97.14 & 2.86 \\
1998 & 97.13 & 2.87 \\
1999 & 97.2 & 2.8 \\
2000 & 97.2 & 2.8 \\
2001 & 97.06 & 2.94 \\
2002 & 97.06 & 2.94 \\
2003 & 96.99 & 3.01 \\
2004 & 97.03 & 2.96 \\
\hline
\end{tabular}

Fuente: Cálculos propios.

Según esta última clasificación regional, el centro ha aumentado ligeramente su participación, al estimular una constante aglomeración. Aunque a menudo se anunció que la liberalización comercial traería un paliativo profundo a las disparidades regionales, el panorama sigue siendo heterogéneo y complejo. La dualidad expresada como centro-periferia o norte-sur, que dividía el núcleo industrial y una periferia agrícola, ha existido históricamente y aún continúa. El centro se ha caracterizado por ser una economía altamente desarrollada e industrializada comparada con el sur. Esta dualidad es una muestra de la persistencia en las disparidades que presentan un carácter regional y geográfico muy claro, en el cual el sur siempre ha permanecido rezagado.

Domingues y Machado Ruiz (2005) encuentran evidencia de que la dispersión industrial en Brasil, como en otros países en desarrollo, cuya característica común ha sido la concentración espacial, se ha limitado a algunas regiones. De forma similar, la anunciada dispersión industrial de la manufactura mexicana es limitada y restringida, sólo los núcleos industriales tradicionales y los estados nuevamente industrializados han tendido a la igualación; la localización y la relocalización se han dado entre los 
estados del centro más que en los de la periferia. Por tanto, al indicar que el movimiento significativo del DF hacia el norte fue la gran contribución de la liberalización y de la integración comercial a la convergencia geográfica de la producción en México, sus virtudes en términos de reducir las diferencias regionales y de promover el desarrollo espacial fueron sobrestimadas. La base de esta afirmación parte de dos aspectos: por un lado, en muchas ocasiones el sur fue dejado de lado en el análisis; por otro, la dispersión venía ocurriendo antes de la liberalización comercial y es probable que otras fuerzas estuvieran en acción para ese entonces.

El centro se ha vuelto más homogéneo, pero aun cuando se han presentado arreglos locacionales que hacen que la geografía industrial luzca más uniforme, el patrón dual también está sujeto a la controversia debido a la existencia de diferencias intrarregionales. El paisaje económico dentro del centro o de la periferia puede incluir así una mezcla de estructuras económicas diversas al interior de estas amplias regiones.

\section{Un contexto multidimensional de la geografía manufacturera} como escenario alternativo

Una vez que permitimos la posibilidad de escenarios alternos los datos muestran la existencia de lo que podríamos denominar una estructura geográfica con multiconglomerados o, en otros términos, una estructura policéntrica con numerosos centros industriales y periferias. Es decir, existe heterogeneidad y fragmentación geográfica en la manufactura mexicana. Hay tres núcleos manufactureros bien definidos: la frontera norte, el Bajío, y el núcleo del centro. Estos contextos multicéntricos hacen difícil caracterizar y resumir la estructura regional al interior de los países. Además los centros múltiples pueden implicar aglomeraciones que están geográficamente separadas una de la otra pero que presentan similares densidades e intensidades en la producción.

\section{Conclusiones}

En este documento hemos encontrado que mientras la reorientación de la política económica tuvo algún efecto, ya con anterioridad se estaba dando una nueva configuración geográfica. A pesar de los cambios en la localización industrial, las grandes y sostenidas diferencias regionales continúan y tienen un impacto sobre el desarrollo en un sentido más amplio. La conclusión general es que el ajuste lento que ha presentado la manufactura no fue desatado en realidad por el programa de liberalización económica como tal. El programa de liberalización y la estrategia exportadora no generaron 
la relocalización y el crecimiento de la importancia relativa del norte y el Bajío, pero reforzaron un proceso ya en marcha. Los datos señalan que hubo un cambio poco significativo en el nivel de aglomeración al hacer las disparidades geográficas continuas. De ahí que se puedan extraer estas conclusiones mas especificas:

1. Desde una perspectiva dinámica ha habido una tendencia hacia la dispersión de la manufactura, pero ésta ha sido mínima.

2. Desde el punto de vista estático, la actividad manufacturera aún está muy aglomerada.

Como la relocalización y la relativa dispersión estaban en proceso, no pueden ser atribuidas completamente a las reformas económicas aunque sí parecen haber tenido algún impacto. La caída más acentuada entre 1980 y 1985 puede representar el ajuste a los primeros esfuerzos por liberalizar el comercio exterior. La segunda etapa significativa fue la entrada al GATT en 1986 y la consolidación del programa de liberalización, pero no podemos verificar los efectos de este periodo. Finalmente, hubo otra disminución de la aglomeración después de TLCAN, mayor que el promedio pero tampoco muy sustancial. Por tanto, las reformas económicas contribuyeron a los cambios locacionales pero el efecto fue limitado en la reducción de la aglomeración. Además, un número de fuerzas, como el libre comercio y la IED, pudo tener sus efectos antes del cambio de política económica. El funcionamiento desigual entre los estados mexicanos sigue siendo por consiguiente una realidad, pues el impacto de la liberalización comercial en el desarrollo regional ha sido insignificante y adverso para los estados del sur.

El rumbo que tome la discusión sobre la dispersión y la descentralización geográfica de la actividad económica también depende de la agrupación regional que consideremos. Aunque los resultados señalan una creciente división centro-periferia y una marcada división norte-sur, las disparidades regionales en la escala intrarregional implican algo más que un simple patrón dual debido a que las diferencias intrarregionales dentro del centro y de la periferia pueden conducir a mayor complejidad territorial industrial. 


\section{Bibliografía}

Bassols Batalla, Ángel, Geografía Económica de México, México, Editorial Trillas, 1998.

Behrens, Kristian, "International Trade and Internal Geography Revisited", Documento presentado en la Third Spring School in Economic Geography, Dijon, Francia, 2003, pp. 1-36.

, Carl Gaigné, Gianmarco Ottaviano y Jacques-François Thisse, Inter-regional and International Trade: Seventy Years After Ohlin, CEPR Discussion Papers, núm. 4065, 2003.

Ben, Denis y Kenneth Hall, Globalisation, a calculus of inequality, Kingston, Ian Randle Publishers, 2002.

Brand, Henk, The Economic Geography Effects of Trade Liberalisation on the National Regions of Spain, European Trade Seminar Group Conference, Nottingham, 2004.

Chamboux-Leroux, Jean Ives, "Efectos de la Apertura Comercial en las Regiones y la Localización Industrial en México", Comercio Exterior, Bancomext, núm. 7 , México, julio de 2001, pp. 600-609.

Corona Jíménez, Miguel Ángel, "Efectos de la Globalización en la Distribución Espacial de las Actividades Económicas", Comercio Exterior, Bancomext, núm. 53, México, enero de 2003, pp. 48-56.

Crozet, Matthieu y Pamina Koening-Soubeyran, Trade Liberalization and the Internal Geography of Countries, 2002, pp. 1-18.

Decuir-Viruez, Luisa, "Institutional Factors in the Economic Growth of Mexico", 43 ERSA Congress 2003 on Peripheries, Centres, and Spatial Development in the New Europe, Finland, University of Jyvaskyla, 2003.

Domingues, Edson Paulo y Ricardo Machado Ruiz, "Industrial cores and peripheries in Brazil", Textos para Discussão CedeplarUFMG td261, Cedeplar, Universidade Federal de Minas Gerais, 2005.

Gordo, Esther, María Gil y Miguel Pérez, "Los Efectos de la Integración Económica sobre la Especialización y Distribución Geográfica de la Actividad Industrial en los Países de la UE", Documento Ocasional, núm. 0303, Madrid, Banco de España, 2003, pp. 1-64.

Hanson, Gordon H., Firms, workers, and the geographic concentration of economic activity, Oxford, Oxford University Press, 2000, pp. 477-494.

"Regional adjustment to trade liberalization", Regional Science and Urban Economics, 28, 1998, pp. 419-444.

Henderson, J. Vernon, Zmarak Shalizi y Anthony Venables, "Geography and development", Journal of Economic Geography, vol. 1, núm. 1, 2001, pp. 81-105.

Jordaan, Jacob y Javier Sánchez-Reaza, Industrial Diversity and Specialisation in Mexican Regions under Free Trade, México, CIDE, 2004, pp. 1-15.

Katz, Isaac M., La apertura comercial y su impacto regional sobre la economía mexicana, México, Miguel Ángel Porrúa Grupo Editorial, 1998.

Klein, Emilio y Víctor Tokman, "Social stratification under tension in a globalized era", CEPAL Review, núm. 72, 2000, pp. 7-29.

Krugman, Paul y Raul Livas Elizondo, "Trade policy and the Third World metropolis", Journal of Development Economics, núm. 49, 1996, pp. 137-150.

y Anthony J. Venables, "Integration, specialization, and adjustment", European Economic Review, vol. 40, núm. 3-5, 1996 , pp. 959-967.

Paluzie, Elisenda, "Trade Policy and Regional Inequalities", Papers in Regional Science and Urban Economics, núm. 80, 2001, 67-85 pp.

Rodríguez-Pose, Andrés y Javier Sánchez-Reaza, Economic Polarization Through Trade, Trade Liberalization and Regional Growth in Mexico, WIDER-United Nations University, 2003.

Sánchez-Reaza, J. y Jacob Jordaan, "Resource Endowments, Externalities and Transport Costs: An Exploration of Alternative Explanations of Regional Production Structures in Mexico", Worshop on Agglomeration and Regional Labour Markets, Berlín, 2002.

Sobrino, Jaime, Competitividad de las ciudades en Mexico, Mexico, El Colegio de México, Centro de Estudios Demográficos, 2003, $619 \mathrm{pp}$.

Venables, Anthony J., Spatial disparities in developing countries: cities, regions and international trade., London School of Economics and Centre for Economic Policy Research, UNU-WIDER, 2003, pp.1-30. 


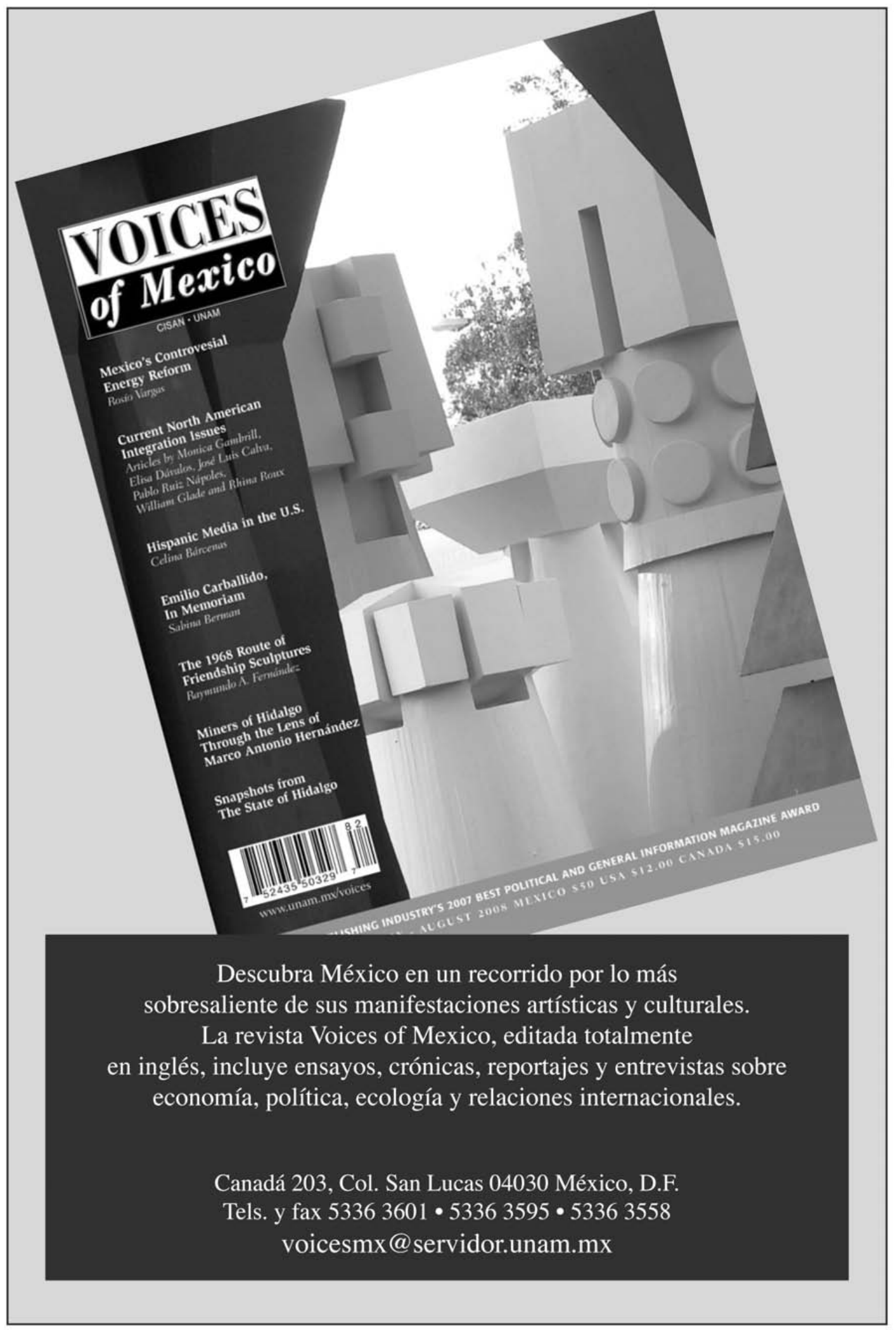

